\title{
IN MEMORY OF ARKADY VIKTOROVICH KRYAZHIMSKIY (1949-2014)
}

\author{
Sergei M. Aseev ${ }^{a, b}$, Alexander G. Chentsov ${ }^{c}$, Alexey A. Davydov ${ }^{b, d, e}$, \\ Nikolai L. Grigorenko ${ }^{e}$, Vyacheslav I. Maksimov ${ }^{c, f}$, \\ Elena A. Rovenskaya ${ }^{b, e}$, Alexander M. Tarasiev ${ }^{c}$ \\ ${ }^{a}$ Steklov Mathematical Institute, Moscow, Russia \\ ${ }^{b}$ International Institute for Applied Systems Analysis, Laxenburg, Austria \\ ${ }^{c}$ Krasovskii Institute of Mathematics and Mechanics, \\ Ural Branch of the Russian Academy of Sciences, Ekaterinburg, Russia \\ ${ }^{d}$ Moscow University of Science and Technology MISiS, Moscow, Russia \\ ${ }^{e}$ Lomonosov Moscow State University, Moscow, Russia \\ ${ }^{f}$ Email: maksimov@imm.uran.ru
}

\begin{abstract}
The article is devoted to the description of Academician Arkady Kryazhimskiy's life path. The facts of the scientific biography of Acad. Kryazhimskiy are presented with the emphasis on his outstanding contribution into the theory of dynamic inversion, the theory of differential games, and control theory. His personal talents in different spheres are also marked out.
\end{abstract}

Key words: Arkady Viktorovich Kryazhimskiy.

Arkady Viktorovich Kryazhimskiy was born on January 2, 1949, in Qingdao, China. In 1971 he graduated from the Department of Mathematics and Mechanics of Gor'kii Ural State University in Sverdlovsk (now, Ekaterinburg) and entered a postgraduate program under the supervision of Yurii Sergeevich Osipov. In 1971 Osipov completed the development of the foundations of positional game theory for control systems with delayed argument and suggested Kryazhimskiy to study an pursuit-evasion differential game for a target set given in an infinite-dimensional phase space of a delay system. That was the time when convex analysis in Hilbert spaces, a division of functional analysis, was actively developed. Kryazhimskiy used technique from this research area to design solution methods for the described problem. He carried out a comprehensive study of an pursuitevasion game with a functional target. Based on the results of these studies, Kryazhimskiy defended his candidate's dissertation "Some Game Problems of Pursuit-Evasion" in 1974.

In July 1972, the Laboratory (later, Department) of Differential Equations, headed by Osipov, was created at the Institute of Mathematics and Mechanics of the Ural Scientific Center of the Russian Academy of Sciences (in Sverdlovsk). Kryazhimskiy worked at this department from its creation till the beginning of the 1990s. In the 1970s, after defending his candidate's dissertation, he abandoned his work on differential games for delayed systems and turned to studying differential games for "ordinary" systems with incomplete information as well as infinite-dimensional control systems. Numerous workshops were held on these topics at the Laboratory of Differential Equations. One of difficult important problems was to extend the basic principles of the theory of positional differential games to "ordinary" systems whose right-hand sides did not satisfy the 
Lipschitz condition in the phase variable. Working on this problem, Kryazhimskiy designed a "universal" implementation of the extremal shift principle, which was independent of the specifics of a control system's phase space. The implementation was based on an interesting idea: if a control system is looked upon as a "control-trajectory" transformation, then the extremal shift rule can be specified in the space of "inputs," i.e., controls, rather than in the space of "outputs," i.e., trajectories. This idea showed a way that later led Kryazhimskiy to the following solution of a differential game for non-Lipschitz "ordinary" systems: passage to the infinite-dimensional functional control space, search for an adequate criterion for the deviation between the "true" and "target" controls, and implementation of extremal shift in terms of this criterion. These results served as a base for Kryazhimskiy's doctoral dissertation "Differential Games for Non-Lipschitz Systems" (1981).

By the beginning of the 1980s, the development of fundamental issues of the theory of positional differential games related to finding general solvability criteria of game problems and describing the general structure of their solutions has been mostly completed. Scientists at the Institute of Mathematics and Mechanics who worked in the area of differential games had to decide on the directions on further studies. One of the possible directions was the development of the theory of positional differential games "into the depth" by designing new solution methods for differential game problems; another, the search for new problems and the development of new theoretical approaches. Kryazhimskiy and his colleagues from the department chose the new research area. They intended to search for topical problems at the interface of subject areas. At that time, along with the studies on control theory and differential games, other research directions were developed successfully at the institute; one of them was concerned with the theory of ill-posed problems. Despite the remoteness of this theory from differential games, specialists within game theory were familiar with the idea of regularization, which played an important role in the theory of ill-posed problems. In particular, the widely known method of positional control with a guide proposed by Nikolai Nikolaevich Krasovskii in the first half of the 1970s was based on the effect of regularization, i.e., elimination of instability in the presence of small information noise. Kryazhimskiy and Osipov set a goal to find an application direction for methods of the theory of differential games in the area of ill-posed problems. Finding a specific direction of applications was an extremely difficult task, since it required problems of a principally new class. In the theory of ill-posed problems, the so-called inverse problems of control systems are closest to objects studied in control theory. A typical inverse problem consists in finding a control implementing a specified trajectory of a system or a given signal from a trajectory. Similar problems in the presence of trajectory perturbations are close to the process of observing a real trajectory of a system generated by an unknown control, which in this case loses the traditional meaning of "control," i.e., rational influence aimed at the optimization of motion, since the control is replaced by an unobservable and uncontrolled "input" fed to the system from the environment. According to the ideology of the theory of ill-posed problems, the unobservable input is to be recovered, and the recovery error must be arbitrarily small for sufficiently small observation error. Since a direct observation of perturbing inputs, as a rule, was not possible, a new problem of dynamic inversion arose, which consisted in the real-time recovery of current values of unobservable inputs from an available signal about the trajectory. Later, the problem of dynamic inversion became an "inversion block" in the general "inversioncontrol" scheme, in which, in the process of operation of a control system exposed to the action of unobservable inputs, current values of inputs are recovered approximately in real time from current, generally speaking, inaccurate observations of states of the system (the "inversion block"); these values, together with the results of direct observations of the system's states are fed to an automatic regulator, which produces current values of the control parameter (the "control block").

In the problem of dynamic inversion, an important requirement on the solution algorithm is its dynamic property, i.e., the real-time mode of operation. With reference to the theory of ill-posed problems, this requirement restricts the class of admissible regularizing algorithms, and the problem 
of dynamic inversion can be referred to as a dynamic regularization problem. Kryazhimskiy and Osipov proposed a new methodological approach to dynamic regularization, which became known as the principle of regularized extremal shift. It is based on the procedure of control with a guide from the theory of positional differential games and consists in the following. The process of dynamic recovery of an unobservable input is interpreted as the process of control of an auxiliary dynamic system (model). The model, which is often a copy of the original system, is essentially different from the latter in that it is controllable: the uncontrolled input is replaced by a control parameter. Current values of the model control are formed in real time by means of the feedback principle, as a reaction to the "real" (inaccurate) information on the current state of the original system and to the accurate information on the current state of the model. The feedback in the control loop of the model is chosen so that the implementation of the model control as a function of time track accurately enough the implementation of the input of the original system.

The described scheme was developed initially for "ordinary" finite-dimensional systems affine in the input variable. For such systems, the choice of a model feedback guaranteeing the proximity of trajectories of the model and of the system was not difficult: it was sufficient to use the standard rule of extremal shift of the model's current state toward the current signal about the system's state. The crucial step, which shaped the further development of the approach, was the understanding that an appropriate regularization of the extremal shift rule provides the required much stronger property - the proximity of the model control to the input of the system in the mean-square metric. The proposed regularization involves the combination of the basic criterion - the extremal shiftwith an auxiliary criterion - the minimum criterion for the norm of the current value of the control parameter. The basic version of the method includes the auxiliary criterion by adding to the main, linear, shift criterion a quadratic smoothing function multiplied by a small regularization parameter. The regularized extremal shift, which consists in the minimization of the resulting criterion in the control variable, corresponds exactly to the application of Tikhonov's regularization method to the extremal shift method. Thus, at the interface of the theory of ill-posed problems and the theory of positional control, a new range of problems was found - dynamic regularization problems - and an approach to their solution was proposed - the method of regularized extremal shift.

The studies on the dynamic inversion of "ordinary" finite-dimensional systems carried out by Kryazhimskiy mainly in the 1980s were summarized in the famous monograph, which presents to the reader a deep theory covering a wide range of issues, from the formulation of dynamic inversion problems, investigation of their solvability, and comparison of the possibilities of dynamic and a posteriori methods to the construction of optimal algorithms and detailed implementation of the "inversion-control" scheme, which played an important motivating role at the initial stage of research. The theory is based on the methods of regularized extremal shift, which combine, as mentioned above, approaches from the theory of positional differential games and the theory of ill-posed problems. Certain divisions of the theory involve methods of the theory of differential equations, control theory (in particular, the techniques of generalized controls), estimation theory, functional analysis, convex analysis, and function approximation theory. Explicit descriptions of algorithms of inversion and inversion-control, which are ready for immediate application and are accompanied by accuracy estimates, are combined with the study of delicate theoretical issues, such as regularizability, order optimality, asymptotic optimality, etc.

In the process of creating the theory of dynamic inversion, its authors developed a new approach to the investigation of some divisions of the theory of solution of operator equations, function approximation theory, etc. One of the strongest developments concerned the application of the dynamic inversion ideology to problems of the classical infinite-dimensional optimization. Studies in this direction began in the second half of the 1980s, when a new iterative algorithm for solving a linear-convex problem of optimal control under phase constraints was proposed. The algorithm was based on the principle of regularized extremal shift applied to an artificially designed dynamic 
model with discrete time. An original optimal control problem is interpreted as an optimization problem in the space of artificially created processes under constraints of the type of equality (provided by the equation of the system) and inclusion (provided by the original phase constraints and the original constraints on the control). A special Lyapunov functional related to the Tikhonov regularization method was introduced and stabilized by means of extremal shift; as a result, the model's states converge to the required solution. In further studies, similar algorithmic schemes, based on regularization ideas, were refined and extended to various classes of extremal problems. This series of papers was mainly concerned with the advance into the area of methods of global nonconvex optimization. One of the papers was devoted to studying a wide class of nonconvex optimization problems with constraints; for these problems, the regularized extremal shift principle produces a converging iterative solution algorithm. Problems of this class are characterized by a geometric condition on the separability of the graph of the "perturbed optimal value" function.

In the middle of the 1980s, Kryazhimskiy started investigations related to defense projects. Until the collapse of the Soviet Union in 1991, the researchers of the Department of Differential Equations of the Institute of Mathematics and Mechanics who worked in the sector headed by Kryazhimskiy took part in joint studies with their colleagues from NPO Energiya (Korolev, Moscow region) and NPO Avtomatika (Sverdlovsk). These studies were devoted to processes of interaction of dynamic systems under incomplete and varying information.

In the beginning of the 1990s, Kryazhimskiy moved to Laxenburg, Austria, where he started to work at the International Institute of Applied Systems Analysis. Until the end of 2012, he headed the Dynamic Systems Program (later integrated into the Advanced Systems Analysis Program). The systemic, comprehensive, approach to the solution of difficult interdisciplinary problems, which is a sort of trademark of the institute, was natural to Kryazhimskiy to the full extent. His role in the investigation of certain large-scale problems, such as various applied game problems, economic growth modeling, finding optimal ways of sustainable development on the global scale, modeling of innovation market dynamics, optimal gas transportation, etc., cannot be overestimated.

Since 1996, Kryazhimskiy had worked at the Steklov Institute of Mathematics of the RAS: first, as a leading researcher and, from 1997, as a chief researcher. Simultaneously, he had been a lecturer at the Department of Optimal Control of the Faculty of Computational Mathematics and Cybernetics at Moscow State University. His lectures were very popular with the students because of their rich content, informative value, and clarity of presentation. The lecturer's personal charm was of no less importance.

The wide scientific scope and industriousness at the highest intellectual level were Kryazhimskiy's important traits. He was successful at solving problems from the most diverse divisions of mathematics and borderline disciplines. His main motives in choosing new problems were the synthesis of disciplines and rich practical content.

A result of Kryazhimskiy's fruitful work and acknowledgement of his remarkable contribution to the development of Russian science was his election to the Academy of Sciences. He has been a corresponding member of the academy since May 1997 and a full member since May 2006.

A talented person is talented in everything. This popular saying is Kryazhimskiy's best characterization. He had been attracted to music and literature since his childhood, being a brilliant guitar player and an author of poetry and songs. Arkady Viktorovich was a responsive and warm-hearted person. He was open to people and did not dominate because of his position and well-deserved authority. His relatives and colleagues noticed his tact, enthusiasm, polymathy, and amazing willingness to both share his ideas and appreciate and discuss ideas of other people.

All who knew him were shocked to receive the news of Arkady Viktorovich's untimely death on 3rd of November 2014.

The International Conference in memory of Arkady Viktorovich Kryazhimskiy was organized by the Krasovskii Institute of Mathematics and Mechanics and the Ural Federal University. The 
conference named "System analysis: modeling and control" was held in Ekaterinburg in October 2016 to get together the colleagues of Kryazhimskiy from different countries for discussing actual scientific problems. The event was a success: more than fifty participants, more than thirty plenary reports. Several selected papers presented at the conference are published in this issue of the journal.

\section{KRYAZHIMSKIY'S MAIN SCIENTIFIC PAPERS}

1. A.V. Kryazhimskii and Yu.S. Osipov, Differential-difference game of encounter with a functional target set, J. Appl. Math. Mech. 37 (1), pp. 1-10 (1973).

2. A.V. Kryazhimskii, A differential-difference game of evasion from a functional target, Izv. Akad. Nauk SSSR, Ser. Tekhn. Kibernet., No. 4, pp. 71-79 (1973).

3. A.V. Kryazhimskii, Some Game Problems of Pursuit-Evasion, Candidates Dissertation in Physics and Mathematics (Sverdlovsk, 1974).

4. A.V. Kryazhimskii, Differential games of pursuit in conditions of imperfect information about the system, Ukr. Math. J. 27 (4), pp. 425-429 (1975).

5. A.V. Kryazhimskii, On the admissibility of an optimal strategy, in Differential Games and Control Problems: Collection of Papers (UNTs AN SSSR, Sverdlovsk, 1975), Issue 15, pp. 125-130 [in Russian].

6. A.V. Kryazhimskii, An alternative in a linear pursuit-evasion game with incomplete information, Dokl. Akad. Nauk SSSR 230 (4), pp. 773-776 (1976).

7. A.V. Kryazhimskii and S.D. Filippov, On a game problem on the convergence of two points on a plane under incomplete information, in Control Problems with Incomplete Information (IMM UNTs AN SSSR, Sverdlovsk, 1976), Issue 19, pp. 62-77 [in Russian].

8. A.V. Kryazhimskii, On the problem of the deviation of a linear system with aftereffect from a functional target, in Game Problems of Control: Collection of Papers (IMM UNTs AN SSSR, Sverdlovsk, 1977), Issue 24, pp. 46-52 [in Russian].

9. A.V. Kryazhimskii, On the theory of positional differential games of pusruit-evasion. Dokl. Akad. Nauk SSSR 239 (4), pp. 779-782 (1978).

10. A.V. Kryazhimskii, On stochastic approximation in differential games, Sov. Math. Dokl. 19, pp. 955-959 (1978).

11. A.V. Kryazhimskii and V.I. Maksimov, Approximation in linear differencedifferential games, J. Appl. Math. Mech. 42 (2), pp. 212-219 (1978).

12. Yu.S. Osipov, A.V. Kryazhimskii, and S. P. Okhezin, Control problems in systems with distributed parameters, in Dynamics of Control Systems: Proceedings of the Third AllUnion Chetaev Conference, Irkutsk, Russia, 1977 (Nauka, Novosibirsk, 1979), pp. 199-208 [in Russian].

13. A.V. Kryazhimskii, Differential Games for Non-Lipschitz Systems, Doctoral Dissertation in Physics and Mathematics (Sverdlovsk, 1980).

14. A.V. Kryazhimskii, On some stable bridges for linear controlled systems, in Optimal Control of Systems with Uncertain Information: Collection of Papers (UNTs AN SSSR, Sverdlovsk, 1980), pp. 35-41 [in Russian]. 
15. A.V. Kryazhimskii, On stable position control in differential games, J. Appl. Math. Mech. 42 (6), pp. 1055-1060 (1980).

16. A.V. Kryazhimskii, Deviation of a linear system with aftereffect from a functional target, J. Dynamic Systems Measurement Control 103 (2), pp. 43-48 (1981).

17. A.V. Kryazhimskii, Game evasion problem for a partially continuous system, in Control and Estimation in Dynamical Systems: Collection of Papers (UNTs AN SSSR, Sverdlovsk, 1982), pp. 25-41 [in Russian].

18. Yu.S. Osipov and A.V. Kryazhimskii, On the dynamic solution of operator equations, Sov. Math. Dokl. 27, pp. 382-386 (1983).

19. A.V. Kryazhimskii and Yu.S. Osipov, Modelling of a control in a dynamic system, Engrg. Cybernetics 21 (2), pp. 38-47 (1984).

20. A.V. Kriazhimskii, V.I. Maksimov, and Iu.S. Osipov, On positional simulation in dynamic systems, J. Appl. Math. Mech. 47 (6), pp. 709-714 (1985).

21. O. Abdyrakhmanov and A.V. Kryazhimskii, On the question of the well-posedness of an optimal control problem, Differents. Uravneniya 20 (10), pp. 1659-1665 (1984).

22. O. Abdyrakhmanov and A.V. Kryazhimskii, On regularization of an optimal control problem for a system with nonuniqueness, Izv. Akad. Nauk TSSR, Ser. Fiz.-Tekh. Khim. Geol. Nauk, No. 4, pp. 3-6 (1984).

23. O. Abdyrakhmanov and A.V. Kryazhimskii, On regularization of an optimal control problem for a system with nonuniqueness. II, Izv. Akad. Nauk TSSR, Ser. Fiz.-Tekh. Khim. Geol. Nauk, No. 6, pp. 7-11 (1984).

24. A.V. Kryazhimskii and Yu.S. Osipov, On positional calculation of $\Omega$-normal controls in dynamical system, Probl. Control Inform. Theory 13 (6), pp. 425-436 (1984).

25. A.V. Kim and A.V. Kryazhimskii, Dynamics of the running point of a trajectory with variable initial condition, in Problems of Control and Modeling in Dynamical Systems: Collection of Papers (UNTs AN SSSR, Sverdlovsk, 1984), pp. 19-27 [in Russian].

26. A.V. Kryazhimskii and Yu.S. Osipov, Modeling of parameters of a dynamic system, in Problems of Control and Modeling in Dynamical Systems: Collection of Papers (UNTs AN SSSR, Sverdlovsk, 1984), pp. 47-68 [in Russian].

27. A.V. Kryazhimskii and Yu.S. Osipov, Best approximation of the differentiation operator in the class of nonanticipatory operators, Math. Notes 37 (2), pp. 109-114 (1985).

28. Yu.S. Osipov and A.V. Kryazhimskii, The Lyapunov function method in a motion simulation problem, in Motion Stability: Collection of Papers (Nauka, Novosibirsk, 1985), pp. 53-56 [in Russian].

29. M.S. Gabrielyan and A.V. Kryazhimskii, The convergence-evasion differential game with $\mathrm{m}$ goal sets, Sov. Math. Dokl. 33, pp. 691-694 (1986).

30. A.V. Kryazhimskii and K.E. Lovtskii, Weak continuity of motions with respect to control for controllable differential inclusions and systems with discontinuous right-hand side, Differents. Uravneniya 22 (11), pp. 1895-1905 (1986). 
31. Yu.S. Osipov and A.V. Kryazhimskii, Positional modeling of a stochastic control in dynamical systems, in Stochastic Optimization: Proceedings of the International Conference, Kiev, Ukraine, 1984 (Springer, Berlin, 1986), Ser. Lecture Notes in Control and Information Sciences 81, pp. 696-704.

32. A.V. Kryazhimskii, On the positional regularizing algorithms for control dynamical systems, in Differential Equations and Applications (Angel Kanchev Univ., Ruse, 1987), pp. 767-770.

33. A.V. Kryazhimskii, Optimization of the ensured result for the dynamical systems, in Proceedings of the International Congress of Mathematicians, Berkeley, CA, USA, 1986 (Amer. Math. Soc., Providence, RI, 1987), Vol. 2, pp. 1171-1179.

34. A.V. Kryazhimskii and Yu.S. Osipov, On the regularization of a convex extremal problem with inexactly given constraints. Application to an optimal control problem with phase constraints, in Some Methods of Positional and Program Control: Collection of Research Papers (UNTs AN SSSR, Sverdlovsk, 1987), pp. 34-54 [in Russian].

35. A.V. Kryazhimskii and Yu.S. Osipov, Inverse problems of dynamics and controllable models, in Mechanics and Scientific - Technological Progress, Vol. 1: General and Applied Mechanics (Nauka, Moscow, 1987), pp. 196-211 [in Russian].

36. A.V. Kryazhimskii and Yu.S. Osipov, Stable solutions of inverse problems of the dynamics of controlled systems, Proc. Steklov Inst. Math. 185, pp. 143-164 (1988).

37. A.V. Kryazhimskii and Yu.S. Osipov, On the methods of positional modeling of control in dynamical systems, in Qualitative Questions in the Theory of Differential Equations and Control Systems: Collection of Research Papers (UNTs AN SSSR, Sverdlovsk, 1988), pp. 3444 [in Russian].

38. A.V. Kryazhimskii and Yu.S. Osipov, On a stable positional recovery of control from measurements of a part of coordinates, in Some Problems of Control and Stability: Collection of Papers (UNTs AN SSSR, Sverdlovsk, 1989), pp. 33-47 [in Russian].

39. A.V. Kryazhimskii, On the continuity of Lebesgue sets in an optimal control problem, in Optimization and Stability Problems in Control Systems: Collection of Research Papers (UNTs AN SSSR, Sverdlovsk, 1990), pp. 54-73 [in Russian].

40. A.Yu. Vdovin and A.V. Kryazhimskii, On the recovery the perturbation set from measurements of the trajectory, in Studies in System Analysis and Applications: Collection of Research Papers (Izd. Ural. Gos. Univ., Sverdlovsk, 1990), pp. 15-35 [in Russian].

41. A.V. Kryazhimskii, The problem of optimization of the ensured result: Unimprovability of full-memory strategies, in Constantin Carath'eodory: An International Tribute (World Sci., Teaneck, NJ, 1991), Vol. 1, pp. 636-675.

42. A.Yu. Vdovin and A.V. Kryazhimskii, On a lower estimate for a positional regularization method for a disturbance recovery problem, in Modeling and Optimization Problems: Collection of Research Papers (UNTs AN SSSR Sverdlovsk, 1991), pp. 3-13 [in Russian].

43. A.V. Kryazhimskii, Dynamical regularizibility of inverse problems for control systems, in System Modelling and Optimization: Proceedings of the 15th IFIP Conference, Zurich, Switzerland, 1991 (Springer, Berlin, 1992), Ser. Lecture Notes in Control and Information Sciences 180, pp. 384-393. 
44. A.V. Kryazhimskii, Order optimal real-time observers for completely observable control systems, Appl. Math. Comput. Sci. 2 (1), pp. 149-154 (1992).

45. A.V. Kryazhimskiy and V.B. Savinov, The traveling-salesman problem with moving objects, J. Comput. Syst. Sci. Int. 33 (3), pp. 144-148 (1993).

46. A.V. Kryazhimskii, Conditions for stable nonanticipatory motion approximation, Proc. Steklov Inst. Math. 211, pp. 221-233 (1995).

47. A.V. Kryazhimskii and Yu.S. Osipov, On differential-evolutionary games, Proc. Steklov Inst. Math. 211, pp. 234-261 (1995).

48. A.V. Kryazhimskii and V.B. Savinov, On a model of conflict interaction with aftereffect in controls, in RoutingDistributional Problems: Collection of Papers (UGTU, Ekaterinburg, 1995), pp. 44-53 [in Russian].

49. A.V. Kryazhimskii, V.I. Maksimov, and E. A. Samarskaia, On Estimation of Forcing Functions in Parabolic Systems: IIASA Working Paper WP-95-75 (IIASA, Laxenburg, 1995).

50. G. Hutschenreiter, Yu.M. Kaniovski, and A.V. Kryazhimskii, Endogenous Growth, Absorptive Capacities and International R\&D Spillovers: IIASA Working Paper WP-95-92 (IIASA, Laxenburg, 1995).

51. A. Kryazhimskii, An Endogenous Growth Model for Technological LeadingFollowing: An Asymptotical Analysis: IIASA Working Paper WP-95-93 (IIASA, Laxenburg, 1995).

52. Yu.S. Osipov and A.V. Kryazhimskii, Inverse Problems for Ordinary Differential Equations: Dynamical Solutions (Gordon and Breach, London, 1995).

53. Yu.S. Osipov, A.V. Kryazhimskii, and V.I. Maksimov, Dynamical inverse problems for systems with distributed parameters, J. Inverse Ill-Posed Probl. 4 (4), pp. 267-282 (1996).

54. V.I. Heymann and A.V. Kryazhimskii, On finite-dimensional parametrizations of attainability sets, Appl. Math. Comput. 78 (2-3), pp. 137-151 (1996).

55. A.V. Kryazhimskii, V.I. Maksimov, A.A. Solov'ev, and A.G. Chentsov, On a probabilistic approach to the quantitative description of the dynamics of natural processes, Problem. Control Inform., Nos. 1-2, pp. 192-210 (1996).

56. A.V. Kryazhimskii, V.I. Maksimov, and Yu.S. Osipov, Reconstruction of Boundary Sources through Sensor Observations: IIASA Working Paper WP-96-97 (IIASA, Laxenburg, 1996).

57. A.V. Kryazhimskii, V.I. Maksimov, and E.A. Samarskaya, On reconstruction of inputs in parabolic systems, Mat. Model. 9 (3), pp. 51-72 (1997).

58. Yu.M. Ermoliev, A.V. Kryazhimskii, and A. Ruszczynski, Constraint aggregation principle in convex optimization, Math. Programm., Ser. B, 76 (3), pp. 353-372 (1997).

59. A.V. Kryazhimskii, V.I. Maksimov, and Yu.S. Osipov, Reconstruction of extremal perturbations in parabolic equations, Comp. Math. Math. Phys. 37 (3), pp. 288-298.

60. A.V. Kryazhimskii, Convex optimization via feedbacks, SIAM J. Contr. and Optimiz. 37 (1), 278302 (1998).

61. A.V. Kryazhimskii and A.M. Tarasyev, Equilibrium and Guaranteeing Solutions in Evolutionary Nonzero Sum Games, IIASA Interim Report IR-98-003 (IIASA, Laxenburg, 1998). 
62. A. Kryazhimskii, A. Nentjes, S. Shibayev, and A. Tarasyev, Searching Market Equilibria under Uncertain Utilities, IIASA Interim Report IR-98-007 (IIASA, Laxenburg, 1998).

63. A.V. Kryazhimskii and V.I. Maksimov, An iterative procedure for solving a control problem with phase constraints, Comp. Math. Math. Phys. 38 (9), pp. 1423-1428 (1998).

64. B.V. Digas, Yu.M. Ermoliev, and A.V. Kryazhimskii, Guaranteed Optimization in Insurance of Catastrophic Risks, IIASA Interim Report IR-98-082 (IIASA, Laxenburg, 1998).

65. A.F. Kleimenov and A.V. Kryazhimskii, Normal Behavior, Altruism and Aggression in Cooperative Game Dynamics, IIASA Interim Report IR-98-076 (IIASA, Laxenburg, 1998).

66. A.V. Kryazhimskii and Yu.S. Osipov, Approximate linear reduction in guidance and evasion differential game, Proc. Steklov Inst. Math. 220, pp. 170-191 (1998).

67. A.V. Kryazhimskii and Yu.S. Osipov, On two-dimensional reduction in a differential game of quality, Proc. Steklov Inst. Math. 224, pp. 198-211 (1999).

68. A.V. Kryazhimskii, A. Nentjes, S.V. Shibayev, and A.M. Tarasyev, A game model of negotiations and market equilibria, J. Math. Sci. 100 (6), pp. 2601-2612 (2000).

69. V.F. Borisov, G. Hutschenreiter, and A.V. Kryazhimskii, Asymptotic growth rates in knowledgeexchanging economies, Ann. Oper. Res. 89, pp. 61-73 (1999).

70. F. Kappel, A. Kryazhimskii, and V. Maksimov, Constraint aggregation principle in the problem of optimal control of distributed parameter systems, in Nonsmooth and Discontinuous Problems of Control and Optimization: Proceedings of the International IFAC Workshop, Chelyabinsk, Russia, 1998 (IIASA, Laxenburg, 1999), pp. 137-141.

71. A.V. Kryazhimskii and G. Sonnevend, Dynamics for bimatrix games via analytic centers, in Dynamics and Control (Gordon and Breach, London, 1999), Ser. Stability and Control: Theory, Methods, and Applications, Vol. 9, pp. 129-138.

72. Yu.M. Kaniovski, A.V. Kryazhimskii, and H. P. Young, Adaptive dynamics in games played by heterogeneous populations, Games Econom. Behav. 31 (1), pp. 50-96 (2000).

73. F. Kappel, A.V. Kryazhimskii, and V.I. Maksimov, Dynamic reconstruction of states and guaranteeing control of a reactiondiffusion system, Dokl. Math. 61 (1), pp. 143-145 (2000).

74. V. Borisov, G. Feichtinger, and A. Kryazhimskii, Optimal enforcement on a pure sellers market of illicit drugs, J. Optim. Theory Appl. 106 (1), pp. 1-22 (2000).

75. Yu.S. Osipov, A.V. Kryazhimskii, and V.I. Maksimov, Dynamic inverse problems for parabolic systems, Differential Equations 36 (5), pp. 643-661 (2000).

76. A.V. Kryazhimskii and Yu.S. Osipov, On an algorithmic criterion of the solvability of game problems for linear controlled systems, Proc. Steklov Inst. Math., Suppl. 1, pp. S154-S162 (2000).

77. A.V. Kryazhimskii and B.V. Digas, Insurance optimization for catastrophic risks: a guarantee approach, in Information Technologies in Economics: Theory, Models, and Methods (Izd. Ural. Gos. Ekon. Univ., Yekaterinburg, 2000), pp. 98-105 [in Russian].

78. S.M. Aseev, A.V. Kryazhimskii, and A.M. Tarasyev, The Pontryagin maximum principle and transversality conditions for an optimal control problem with infinite time interval, Proc. Steklov Inst. Math. 233, pp. 64-80 (2001). 
79. A.V. Kryazhimskii and S.V. Paschenko, On the problem of optimal compatibility, J. Inverse Ill-Posed Probl. 9 (3), pp. 283300 (2001).

80. A.V. Kryazhimskii and A. Ruszczynski, Constraint aggregation in infinite-dimensional spaces and applications, Math. Oper. Res. 26 (4), pp. 769-795 (2001).

81. A.V. Kryazhimskii, Optimization problems with convex epigraphs. Application to optimal control, Intern. J. Appl. Math. Comput. Sci. 11 (4), pp. 773-801 (2001).

82. A. Kryazhimskii, A. Nentjes, S. Shibayev, and A. Tarasyev, Modeling market equilibrium for transboundary environmental problem, Nonlinear Anal. 47 (2), pp. 991-1002 (2001).

83. A.V. Kryazhimskii, Optimization problems with convex epigraphs. Application to optimal control, Intern. J. Appl. Math. Comput. Sci. 11 (4), pp. 773-801 (2001).

84. A.V. Kryazhimskii and S.V. Pashchenko, On the solution of the linear time-optimal control problem with mixed constraints, J. Math. Sci. 114 (3), pp. 1345-1362 (2003).

85. A. Kryazhimskii, C. Watanabe, and Y. Tou, Dynamic model of market of patents and equilibria in technology stocks, Comput. Math. Appl. 44 (7), pp. 979-995 (2002).

86. A.V. Kryazhimskii and Yu.S. Osipov, Extremum problems with separable graphs, Cybern. Syst. Anal. 38 (2), pp. 175-194 (2002).

87. G. Klaassen, A. Kryazhimskii, O. Nikonov and Ya. Minullin, On a game of gas pipeline projects competition, in Game Theory and Applications: Proceedings of the ICM 2002 Satellite Conference, Qingdao, China, 2002 (Qingdao Publ., Qingdao, 2002), pp. 327-334.

88. S.M. Aseev, A.V. Kryazhimskii and G. Hutschenreiter, A dynamic model of optimal investment in research and development, in Modern Mathematics and Applications (Inst. Kibernet. AN Gruzii, Tbilisi, 2005), Vol. 9, pp. 3-43 [in Russian].

89. A.V. Kryazhimskiy and C. Watanabe, Optimization of Technological Growth (Gendaitosho, Kanagawa, 2004).

90. A.V. Kryazhimskii and R.A. Usachev, On a convex two-level optimization problem, in Nonlinear Dynamics and Control: Collection of Papers (Fizmatlit, Moscow, 2004), Issue 4, pp. 257-286 [in Russian].

91. A.V. Kryazhimskiy and V.I. Maksimov, A solution algorithm for problems of optimal control in Hilbert spaces, J. Math. Sci. 121 (2), pp. 2226-2247 (2004).

92. A. Kryazhimskiy and V. Maksimov, On exact stabilization of an uncertain dynamical system, J. Inverse Ill-Posed Probl. 12 (2), pp. 145-182 (2004).

93. A.V. Kryazhimskii and Yu.S. Osipov, The method of extremal shift and optimization problems, Proc. Steklov Inst. Math., Suppl. 2, pp. S91-S114 (2004).

94. G. Klaassen, A.V. Kryazhimskii and A. M. Tarasyev, Multiequilibrium game of timing and competition of gas pipeline projects, J. Optim. Theory Appl. 120 (1), pp. 147-179 (2004).

95. S.M. Aseev and V. Kryazhimskii, The Pontryagin maximum principle for an optimal control problem with a functional specified by an improper integral, Dokl. Math. 69 (1), pp. 89-91 (2004). 
96. A. Kryazhimskiy and V. Maksimov, Parallelization in an algorithm of multi-dimensional nonconvex optimization: an application to insurance network design, in Parallel Processing and Applied Mathematics (Springer, Berlin, 2004), Ser. Lecture Notes in Computer Science, Vol. 3019, pp. $754-761$.

97. S.M. Aseev and V. Kryazhimskiy, The Pontryagin maximum principle and transversality conditions for a class of optimal control problems with infinite time horizons, SIAM J. Control Optim. 43 (3), pp. 1094-1119 (2004).

98. A. Kryazhimskiy, Ya. Minullin and L. Schrattenholzer, Global long-term energy-economyenvironment scenarios with an emphasis on Russia, Perspectives in Energy 9, pp. 119-137 (2005).

99. S.A. Brykalov, O.N. Golovina and A.V. Kryazhimskii, Nash equilibrium in multi-player games with the choice of time instants and integral cost functionals, J. Math. Sci. 140 (6), pp. 796-807 (2007).

100. A. Kryazhimskii and V. Maksimov, On identification of nonobservable contamination inputs, Environmental Modelling and Software 20 (8), pp. 1057-1061 (2005).

101. S.M. Aseev, G. Hutschenreiter and A. V. Kryazhimskii, A dynamical model of optimal investment in R\&D, J. Math. Sci. 126 (6), pp. 1495-1535 (2005).

102. A. Kryazhimskiy, A system-robust stabilization technique with application to an uncertain model of global carbon cycle, in Modeling and Control of Autonomous Decision Support Based Systems: Proceedings of the 13th International Workshop on Dynamics and Control, Wiesensteig, Germany, 2005, Ed. by E. Hofer and E. Reithmeier (Shaker, Aachen, 2005), pp. 149-156.

103. S. Aseev, G. Hutschenreiter, A. Kryazhimskiy and A. Lysenko, A dynamic model of optimal investment in research and development with international knowledge spillovers, Math. Comput. Model. Dyn. Syst. 11 (2), pp. 125-133 (2005).

104. A. V. Kryazhimskii and V. I. Maksimov, A solution algorithm for problems of optimal control in Hilberts space, J. Math. Sci. 121 (2), pp. 2226-2247 (2004).

105. Yu.S. Osipov, A.V. Kryazhimskii and E.A. Rovenskaya, An optimal compatibility parameter problem: Constructive regularization method, Izv. Ural. Gos. Univ., No. 10, pp. 128-166 (2006).

106. A.V. Kryazhimskii, O. Nikonov and Ya. Minullin, Game of timing in gas pipeline projects competition: Simulation software and generalized equilibrium solutions, in Advances in Dynamic Games: Applications to Economics, Management Science, Engineering, and Environmental Management (Birkhäuser, Boston, 2006), Ser. Annals of the International Society of Dynamic Games, Vol. 8, pp. 237-252.

107. Yu.S. Osipov and A.V. Kryazhimskii, Problems of dynamic inversion, Herald Russ. Acad. Sci. 76 (4), pp. 352-360 (2006).

108. A.V. Kryazhimskiy and V.I. Maksimov, Dynamical state reconstruction and guaranteeing control for a system of parabolic equations, Proc. Steklov Inst. Math. 253 (Suppl. 1), pp. S168-S184 (2006). 
109. A.V. Kryazhimskiy, Yu.S. Osipov's work in mathematical control theory, Russ. Math. Surv. 61 (4), pp. 593-610 (2006).

110. S.M. Aseev and A.V. Kryazhimskii, The Pontryagin maximum principle and optimal economic growth problems, Proc. Steklov Inst. Math. 257, pp. 1-255 (2007).

111. S.M. Aseev and A.V. Kryazhimskii, On a class of optimal control problems arising in mathematical economics, Proc. Steklov Inst. Math. 262, pp. 10-25 (2008).

112. A.V. Kryazhimskii, V.I. Maksimov, E.A. Rovenskaya and M.V. Rodkin, On a regime of repetition of rare strong events (catastrophes): New approaches and results of their application, in Change in the Environment and Climate: Natural Catastrophes and Induced Technogenic Catastrophes (Inst. Geogr. RAN, Moscow, 2008), Vol. 3, pp. 158-189 [in Russian].

113. B.D. Fath, A.V. Kryazhimskiy, H. Liljenstroem and E. Rovenskaya, Introduction: Towards the design of an integrated socio-environmental assessment model for the Baltic Sea region, in Evolutionary and Deterministic Methods for Design, Optimization and Control: Applications to Industrial and Societal Problems (Int. Center Numer. Meth. Eng., Barcelona, 2008), pp. $425-429$.

114. A.V. Kryazhimskii and R.A. Usachev, Convex two-level optimization problem, Comput. Math. Model. 19 (1), pp. 73-101 (2008).

115. A.V. Kryazhimskiy and V.I. Maksimov, On rough inversion of a dynamical system with a disturbance, J. Inverse Ill-Posed Probl. 16 (6), pp. 587-600 (2008).

116. S.M. Aseev and A.V. Kryazhimskiy, Shadow prices in infinite-horizon optimal control problems with dominating discounts, Appl. Math. Comput. 204 (2), pp. 519-531 (2008).

117. A. Kryazhimskiy, M. Obersteiner and A. Smirnov, Infinite-horizon dynamic programming and application to management of economies effected by random natural hazards, Appl. Math. Comput. 204 (2), pp. 609-620 (2008).

118. Yu.S. Osipov, A.V. Kryazhimskii and V.I. Maksimov, N.N. Krasovskiis extremal shift method and problems of boundary control, Autom. Remote Control 70 (4), pp. 577-588 (2009).

119. A. Kryazhimskiy, On a Boundedly Rational Pareto-Optimal Trade in Emission Reduction, IIASA Interim Report IR-09-017 (IIASA, Laxenburg, 2009).

120. A.V. Kryazhimskii and Yu. S. Osipov, Idealized program packages and problems of positional control with incomplete information, Proc. Steklov Inst. Math. 268 (Suppl. 1), pp. S155S174 (2010).

121. A.V. Kryazhimskii and Yu.S. Osipov, On dynamical regularization under random noise, Proc. Steklov Inst. Math. 271, pp. 125-137 (2010).

122. A.V. Kryazhimskii, Models of economic growth. Authoritarian planning and up-to-theminute solutions, in Problems of Dynamical Control (MAKS, Moscow, 2010), Issue 5, pp. 157-165 [in Russian].

123. A. Kryazhimskiy, On a decentralized boundedly rational emission reduction strategy, in Dynamic Systems, Economic Growth and the Environment, Ed. by J. Crespo-Cuaresma, T. Palokangas, and A. Tarasyev (Springer, Berlin, 2010), Ser. Dynamic Modeling and Econometrics in Economics and Finance, Vol. 12, pp. 215-235. 
124. A.V. Kryazhimskii, S.P. Konovalov, and M.S. Nikolskii, A simplified model of tax collection from enterprises in the presence of legal and shadow capital, Comp. Math. Modeling 24 (3), pp. 378-403 (2010).

125. A. Kryazhimskiy, Two-step win-stay, lose-shift and learning to cooperate in the repeated prisoners dilemma, Int. Game Theory Rev. 12 (4), pp. 437-451 (2010).

126. A. Kryazhimskiy and V. Maksimov, Resource-saving infinite-horizon tracking under uncertain input, Appl. Math. Comput. 217 (3), pp. 1135-1140 (2010).

127. Yu.S. Osipov, A. V. Kryazhimskii, and V. I. Maksimov, Dynamic Recovery Methods for Inputs of Control Systems (UrO RAN, Ekaterinburg, 2011) [in Russian].

128. A.V. Kryazhimskii and V.I. Maksimov, Extremal control methods and dynamical inversion problems, Vestn. Nizhegorod. Univ. 4, pp. 184-185 (2011).

129. A.V. Kryazhimskiy and V.I. Maksimov, Resource-saving tracking problem with infinite time horizon, Differential Equations 47 (7), pp. 1004-1013 (2011).

130. Yu.S. Osipov, A.V. Kryazhimskii, and V.I. Maksimov, Some algorithms for the dynamic reconstruction of inputs, Proc. Steklov Inst. Math. 275 (Suppl. 1), pp. S86-S120 (2011).

131. A.V. Kryazhimskii, Numerical encoding of sampled controls and an approximation metric criterion for the solvability of a guidance game problem, Proc. Steklov Inst. Math. 276 (Suppl. 1), pp. S106-S125 (2012).

132. A.V. Kryazhimskiy and Yu.S. Osipov, On the solvability of problems of guaranteeing control for partially observable linear dynamical systems, Proc. Steklov Inst. Math. 277, pp. 144159 (2012).

133. S.M. Aseev, K.O. Besov and A.V. Kryazhimskii, Infinite-horizon optimal control problems in economics, Russ. Math. Surv. 67 (2), pp. 195-253 (2012).

134. A.V. Kryazhimskii and A.V. Raigorodskaya, On uniform behavior strategies in infinite repeating games, in Problems of Dynamical Control (MAKS, Moscow, 2012), Issue 6, pp. 133-159 [in Russian].

135. A. Frank, M.G. Collins, M. Clegg, U. Dieckmann, V. Kremenyuk, A. Kryazhimskiy, J. Linnerooth-Bayer, S. Levin, A. Lo, B. Ramalingam, J. Ramo, S. Roy, D. Saari, Z. Shtauber, K. Sigmund, J. Tepperman, S. Thurner, W. Yiwei and D. von Winterfeldt, Security in the Age of Systemic Risk: Strategies, Tactics and Options for Dealing with Femtorisks and Beyond, IIASA Interim Report IR-12-010 (IIASA, Laxenburg, 2012).

136. A. Kryazhimskiy and A. Puchkova, Towards Detection of Early Warning Signals on Financial Crises, IIASA Interim Report IR-12-001 (IIASA, Laxenburg, 2012).

137. A.V. Kryazhimskiy, Relaxation of optimal control problems and linearquadratic systems, Dynamics of Continuous, Discrete and Impulsive Systems, Ser. B: Appl. and Algorithms 19 (1-2), pp. 17-42 (2012).

138. A.V. Kryazhimskii and V. I. Maksimov, On combination of the processes of reconstruction and guaranteeing control, Automat. Remote Control 74 (8), pp. 1235-1248 (2013). 